(C) 1977 IEEE. Personal use of this material is permitted. However, permission to reprint/republish this material for advertising or promotional purposes or for creating new collective works for resale or redistribution to servers or lists, or to reuse any copyrighted component of this work in other works must be obtained from the IEEE.

IEEE Transactions on Nuclear Science, Vol.NS-24, No.3, June 1977

\title{
SOME ASPECTS OF THE VACUUM SYSTEM FOR THE PROPOSED STORAGE RING CESR*
}

M. Billing, N. Mistry, L. Phillips, and D. Thomas Laboratory of Nuclear Studies, Corne1l University Ithaca, N. Y. 14853

\section{Summary}

The vacuum system for the proposed $\mathrm{e}^{+} \mathrm{e}^{-}$storage ring CESR must handle the high power-density of synchrotron radiation hitting the walls, and the vacuum chamber must be designed to minimize "highermode" losses due to beam-induced RF fields. We present brief descriptions of some prototype components and results of tests on these prototypes.

The vacuum system for the $e^{+} e^{-}$storage ring CESR ${ }^{1}$ is designed to maintain an average pressure of about $10^{-8}$ Torr in the presence of circulating electron and positron beams of $100 \mathrm{~mA}$ each. The gas load arises mainly from desorption by the intense synchrotron radiation hitting the walls of the extruded aluminum vacuum chamber. The gas will be pumped by distributed sputter-ion pumps inserted in the pump chamber, which is extruded integrally with the beam chamber and the cooling-water channel. Figure 1 shows a prototype pump module protruding from a section of the vacuum chamber. The pump design is similar to that used in SPEAR $^{2}$. Pumping slots are punched in the $4 \mathrm{~mm}$-thick wall between the pump and beam chambers using a specially designed machine capable of punching chambers up to 11 meters long. A single continuous extrusion 8.2 meters long will be used for each halfcell of the normal lattice, which consists of two dipole magnets, a quadrupole and a sextupole. ${ }^{3}$ The pump chamber will be cut away where the extrusion has to fit through the quadrupole and sextupole. This avoids a flanged joint between bend chambers and quadrupole straights, minimizing changes in beamchamber profile.

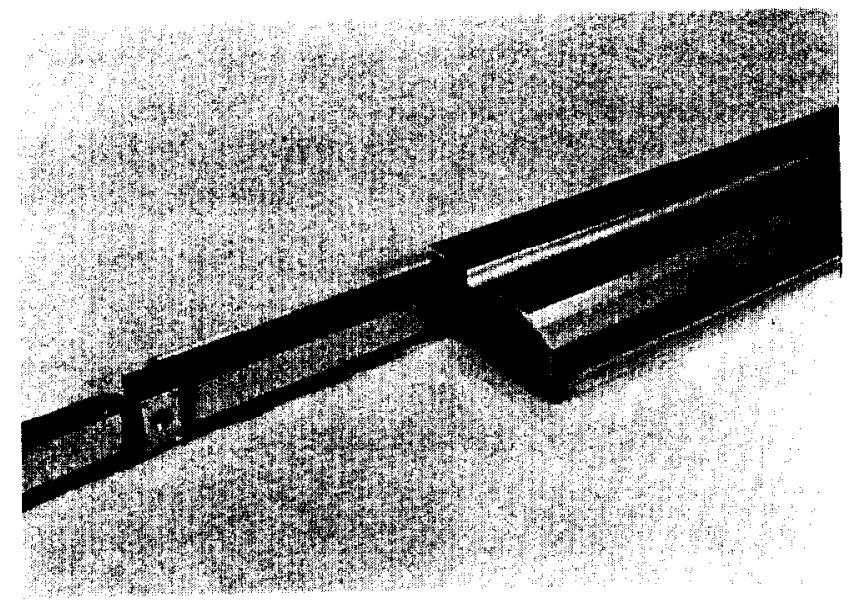

Fig. I CESR Vacuum Chamber and Ion Pump

In order to minimize "higher-mode" losses due to beam-induced RF fields, the beam chambers and all

*Work supported by the National Science Foundation joints, insertions, etc., have to be designed, without sudden steps or cavity-like structures. Figure 2 shows a gate valve modified to operate with a shaped aluminum block following the gate, so that in the "open" position the block is wedged in line with the beam chamber, presenting a smooth unbroken chamber wa11. RF gaskets are used to make positive contact. This valve has operated satisfactorily for over 4000 cycles in a vacuum $\left(10^{-8}\right.$ Torr $)$.

An example of an expansion joint between chambers is shown schematically in Figure 3 . This shows the water-cooled wall of the chamber where the synchrotronradiation (SR) hits. The $8 \mathrm{~mm}$-thick wall is tapered outward, so that the region of the sliding joint is protected from the intense SR. An aluminum "sleeve" slides over the end of the chamber and is attached to the other. An RF gasket at the sliding joint provides good contact between the chambers. A model sliding joint (Figure 4) was used to measure highermode losses using the method of Sands. 4 The power radiated at each such "joint" by two circulating beams of $100 \mathrm{~mA}$ each, in single bunches of gaussian width $2 \sigma=7.44 \mathrm{~cm}$, is expected to be 140 watts. This power is prevented from

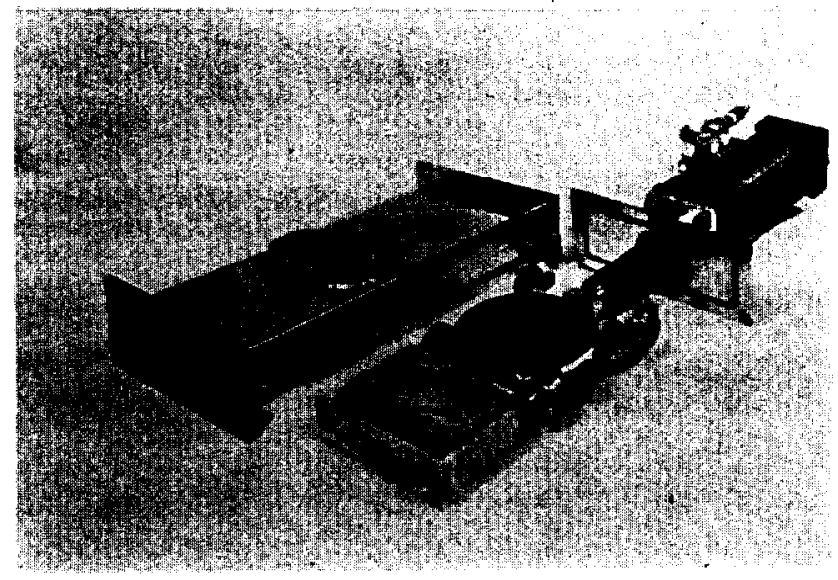

Fig. 2 Modified Gate Valve

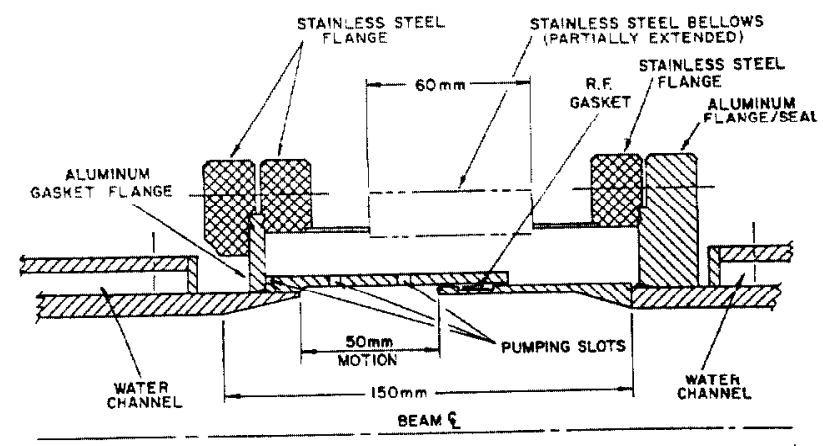

Fig. 3 Expansion Joint Between Chambers 


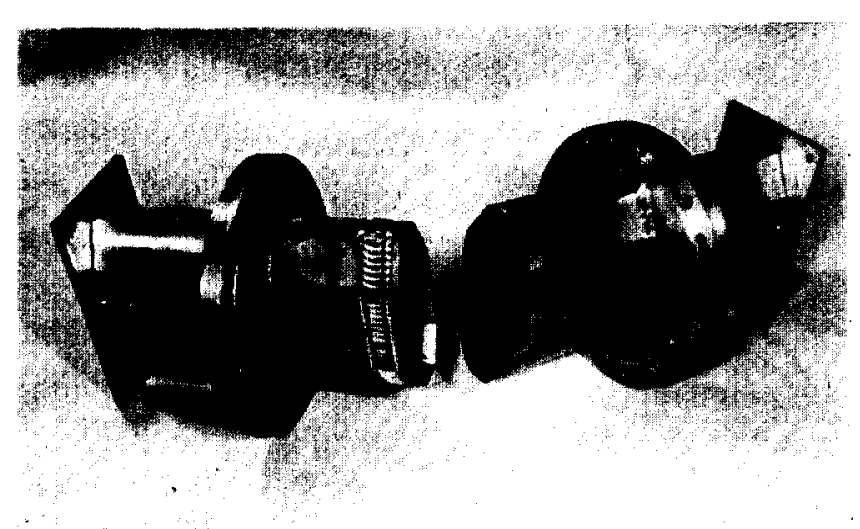

Fig. 4 Mode 7 Sliding Joint

leaking out to the thin stainless steel bellows by the RF gasket. In bench tests at milliwatt RF levels, attenuations of between $10 \mathrm{~dB}$ and $30 \mathrm{~dB}$ have been measured using various types of "finger" contacts. The aluminum contact surfaces on the chamber walls were copper plated for more reliable and consistent contact. Tests are still in progress to determine the optimum joint configuration and gasket material. High-power RF tests were conducted on gaskets in a cylindrical sliding joint model constructed as a resonant S-band cavity at $2856 \mathrm{MHz}$. The configuration was such that peak currents of $\sim 1000 \mathrm{~A}$ would flow across the test joint at pulsed power levels of $200 \mathrm{~kW}$ in the cavity. "Metex" gasket material (woven monel or stainless steel braid) was tested at power levels of $160 \mathrm{~kW}$ (pulsed at 1:4000 duty cycle) for two periods of 17 hours each. No evidence of sparking or burning at the gasket was seen. However, the braid left a residue of fine pieces of wire in the chamber, and is unacceptable for that reason. A similar test was conducted with gold-plated beryllium-copper finger contacts as the gasket material, at $150 \mathrm{~kW}$ levels. No evidence of sparking or burning at the gasket was observed. slight discoloration outside the joint, in the bellows cavity, indicated multipactoring caused by RF leaking through the gasket. Leakage levels (ratios) at $h i g h$ power agreed well with those measured at milliwatt levels (See above).

The complicated design of a sliding joint may be avoided if a simple bellows could be designed such that higher-mode losses are reasonably low. Measurements are in progress on a model elliptical welded bellows having an interior profile matching the beam chamber. Preliminary results indicate that "finetuning" of the bellows design is possible in order to reduce $R F$ losses. The beam pipe is tapered, as shown in Figure 5 , to match the impedance of the bellows. Figure 6 shows the resultant power-loss as a function of the "step", 5 , for a bellows of 23 convolutions, extended to a total length of $7 \mathrm{~cm}$. The step was varied by adding tapered pieces of the same slope. A clear minimum is observed at $s \approx 3 \mathrm{~mm}$. The power loss in this configuration is $\approx 48$ watts for a bunch-length of $2 \sigma=7.44 \mathrm{~cm}$ as before. (Part of this loss is due to resistive losses in the stainless steel bellows surface, and may be reduced by copper-plating). However, calculations indicate a very steep rise of the power loss with decreasing bunch length. Further work is in progress to measure this dependence.

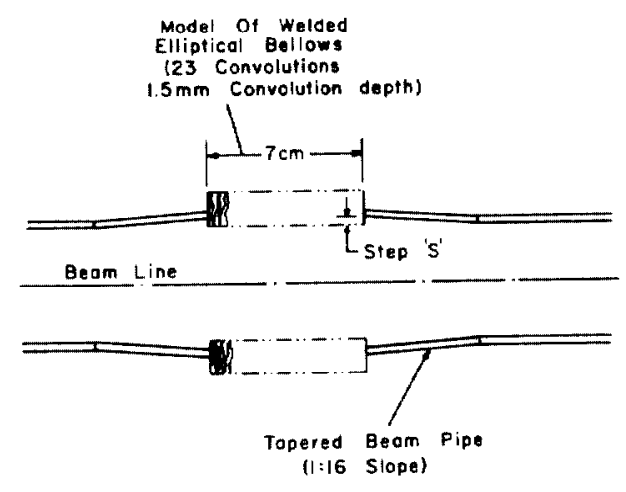

Fig. 5 Elliptical Bellows with Tapered Beam Chamber

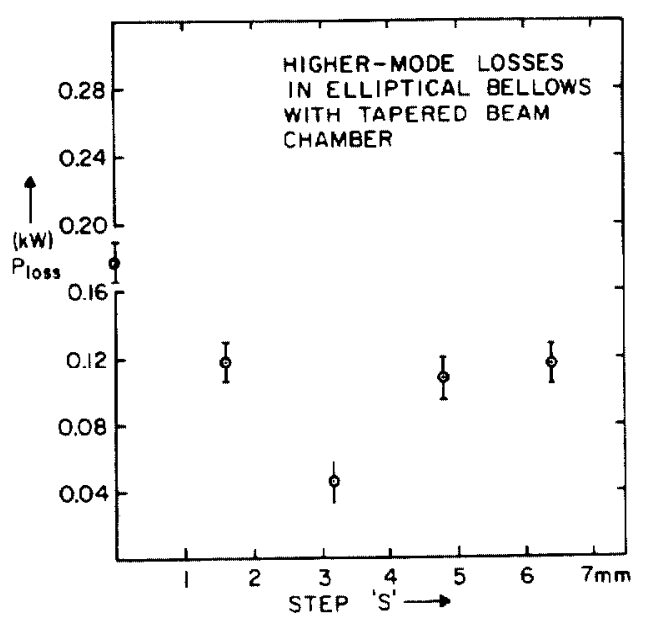

Fig. 6 Power loss at Bellows Joint. Errors are rough estimates of relative error only.

Various techniques are being tried for transitions from the aluminum chambers to the stainless steel flanges and fittings. For tubular transitions of up to $5 \mathrm{~cm}$ diameter, several vacuum brazed transitions and friction-welded transitions have been heat cycled between $20{ }^{\circ} \mathrm{C}$ and $200^{\circ} \mathrm{C}$ for over $800 \mathrm{cycles}$ without failure. For large diameters, we have tried two types of stainless steel knife-edge seals directly on aluminum flanges to be welded on to the vacuum chamber. These are shown as part of Figure 3 . One alternative is to use a thick $(\sim 2.5 \mathrm{~cm})$ aluminum flange bolted up to a standard stainless steel of $8^{\prime \prime}$ $0 . D$. knife-edge flange. The other choice is to capture a thin $(\sim 0.6 \mathrm{~cm})$ aluminum flange between two stainless steel knife-edges. A few flanges of both types were heat cycled between $20^{\circ} \mathrm{C}$ and $200^{\circ} \mathrm{C}$. The thick al uminum flanges fajled to seal after only five
to ten cycles. I. Shen examined under a microscope, it 
was found that the differential expansion and contraction of the two materials had caused the knifeedge to scrape back and forth on the aluminum by about $1.5 \mathrm{~mm}$, with consequent leakage. However, the thin al uminum flanges showed no failures up to 570 cycles. At this point, resealing tests were started on five thin flanges. The flanges were repeatedly disassembled and resealed in turn, with about 20 to 40 heat cycles between tests. Some flanges resealed up to 8 times without failure. Others have leaked after two resealings. It appears difficult to reseal reliably without resurfacing the aluminum flanges. (Small diameter $(5 \mathrm{~cm}$ ) knife-edge seals on aluminum have been successfully used on our prototype apparatus without failure after many resealings). We are investigating the use of the DPI welding technique, pioneered at DESY5, for large diameter stainless steel to aluminum joints between chambers.

We wish to thank Mr. Roger Steinberg for invaluable help in the machining and testing of the prototype apparatus.

\section{References}

1. For parameters of the vacuum system, see CESR Design Report, CLNS-345, October 1976, Laboratory of Nuclear Studies, Cornell University, Ithaca, N. Y. 14853.

2. U. Cummings et al., J. Vac. Sci. Technology $\underline{8}$, $348(1971)$.

3. M. Tigner, "CESR - An Electron Positron Colliding Beam Facility at Cornell", these Proceedings.

4. M. Sands and J. Rees, "A Bench Measurement of the Energy Loss of a Stored Beam to a Cavity," PEP-95, Stanford Linear Accelerator Center, Stanford, California, August 1974.

5. H. Hartwig and J. Kouptsidis, "New Techniques for the PETRA Vacuum System", these Proceedings. 